Article

\title{
Determination of the Main Nucleosides and Nucleobases in Natural and Cultured Ophiocordyceps xuefengensis
}

\author{
Juan Zou ${ }^{1,2}$, Ling $\mathrm{Wu}^{1}{ }^{1}$, Zheng-Mi He ${ }^{1}$, Ping Zhang ${ }^{1}$ and Zuo-Hong Chen ${ }^{1, *}$ \\ 1 College of Life Science, Hunan Normal University, Changsha 410081, China; zoujuantone@126.com (J.Z.); \\ wuling15963@163.com (L.W.); hezhengmi1101@163.com (Z.-M.H.); zhangping0000@163.net (P.Z.) \\ 2 Key Laboratory of Research and Utilization of Ethnomedicinal Plant Resources of Hunan Province, \\ College of Biological and Food Engineering, Huaihua University, Huaihua 418000, China \\ * Correspondence: chenzuohong@263.net; Tel.: +86-731-8887-2557; Fax: +86-731-8888-3310
}

Received: 22 August 2017; Accepted: 9 September 2017; Published: 11 September 2017

\begin{abstract}
Ophiocordyceps xuefengensis, a recently described species of Ophiocordyceps that is associated with the larvae of Phassus nodus (Hepialidae) in the living root or trunk of the medicinal plant Clerodendrum cyrtophyllum, is the largest known Cordyceps species and is recognized as a desirable alternative for natural Ophiocordyceps sinensis. This study investigated the main nucleosides and nucleobases in natural and cultured Ophiocordyceps xuefengensis. The contents of the nucleosides and nucleobases in the natural and cultured samples were determined by reverse phase HPLC. The highest concentration of adenosine was found in the natural fruit body and the cultured stroma, with almost no adenosine in the cadaver of Phassus nodus. The contents of adenine, guanosine, uridine and uracil in the cultured mycelium were significantly higher than those in the natural sample. Inosine was only detected in the natural samples. Thymidine and 2-deoxyadenosine were only found in the cadaver of Phassus nodus. Cordycepin was not detected in the five samples examined. These results suggested that the cultured mycelium and cultured stroma of Ophiocordyceps xuefengensis might be a promising substitute for natural O. xuefengensis.
\end{abstract}

Keywords: Ophiocordyceps xuefengensis; Phassus nodus; stroma; nucleosides

\section{Introduction}

Nucleosides, as pharmaceutical intermediates and/or prodrugs, play an important role in drug development, and as chemotherapeutic agents, they are widely introduced for the medical treatment of cancer [1,2]. Nucleosides and their nucleobases are one of the major active components of Cordyceps [3,4]. Up to now, approximately twenty varieties of nucleosides and their analogues, e.g., cordycepin, adenosine, uridine, guanosine, and inosine, have been found in Cordyceps [2]. Among these, Cordycepin, namely, $3^{\prime}$-deoxyadenosine, is the most notable adenosine analogue from some Cordyceps, showing various pharmacological activities, such as antimicrobial, antitumour, anti-inflammatory and immunomodulation activities [5-7]. Adenosine plays an important role in biochemical processes in organisms, is a major nucleoside in Cordyceps and is recognized as a chemical marker for the quality control of Cordyceps sinensis (Ophiocordyceps sinensis) and its substitutes in China [8]. Therefore, the determination of the content and types of nucleosides is essential for pharmacological studies and the quality control of Cordyceps and its products.

Ophiocordyceps xuefengensis Ting C. Wen et al. a recently described species of Ophiocordyceps parasitized with the larvae of Phassus nodus (Hepialidae) in the living root or trunk of the medicinal plant Clerodendrum cyrtophyllum in the Hunan Province of Southern China, and it is recognized as a 
desirable alternative for natural Ophiocordyceps sinensis [9]. A recent report shows that O. xuefengensis enhances the anti-tumour activity of DC-CIK cells, mainly by promoting proliferation [10].

Recently, the anamorphic state of Ophiocordyceps xuefengensis was isolated from the stroma of the fungus, and the fungus bearing stroma was cultivated successfully in artificial media [11], which provided us with a good opportunity to systematically analyse the nucleoside content of natural and cultured O. xuefengensis. In this study, the natural O. xuefengensis, cultured mycelium, coremium and stroma of $O$. xuefengensis, were systematically analysed for seven nucleosides (cordycepin, adenosine, guanosine, uridine, inosine, thymidine and 2-deoxyadenosine) and two nucleobases (adenine and uracil) by high-performance liquid chromatography coupled with high-resolution mass spectrometry.

\section{Results and Discussion}

\subsection{Calibration Curves, Limit of Detection (LOD), Limit of Quantification (LOQ) and Method Validation}

The linearity, regression, reproducibility and recovery studies and the HPLC profile of nine investigated nucleosides and nucleobases were performed using reference standard samples and are shown in Tables 1 and 2 and Figure 1. The linearity of each standard was confirmed by plotting the peak area ratio of the individual standard versus the concentration of the standard. The correlation coefficients of all the target components exceeded 0.999 , with a good linearity. The LOD and LOQ of the nine analytes were determined at signal-to-noise ratios $(\mathrm{S} / \mathrm{N})$ of 3 and 10 , and the ranges were $0.002-0.01$ and $0.01-0.1 \mu \mathrm{g} / \mathrm{mL}$, respectively. These data indicated that the HPLC analysis of the nucleosides and nucleobases was sensitive for the qualitative and quantitative determination of the components cordycepin, 2-deoxyadenosine, thymidine, adenosine, guanosine, uridine, inosine, adenine and uracil.

Table 1. Linear regression data, limit of detection (LOD), limit of quantification (LOQ) for nine analytes by HPLC.

\begin{tabular}{|c|c|c|c|c|c|}
\hline \multirow{2}{*}{ Analyte } & \multicolumn{3}{|c|}{ Linear Regression Data } & \multirow{2}{*}{$\operatorname{LOD}(\mu \mathrm{g} / \mathrm{mL})$} & \multirow{2}{*}{$\operatorname{LOQ}(\mu \mathrm{g} / \mathrm{mL})$} \\
\hline & Regression Equation & $\mathrm{r}^{2}(n=9)$ & Linear Range $(\mu \mathrm{g} / \mathrm{mL})$ & & \\
\hline Uracil & $Y=8.54854 \times 10^{7} x+19748.4$ & 0.99986 & $0.5-99.0$ & 0.005 & 0.015 \\
\hline Uridine & $Y=5.30058 \times 10^{7} x-6067.1$ & 0.99997 & $0.5-149.3$ & 0.010 & 0.050 \\
\hline Inosine & $Y=4.48713 \times 10^{7} x+4348.1$ & 0.99995 & $0.5-148.5$ & 0.010 & 0.100 \\
\hline Guanosine & $Y=5.31942 \times 10^{7} x-37180.3$ & 0.99902 & $0.5-147.8$ & 0.010 & 0.050 \\
\hline Adenine & $Y=9.81843 \times 10^{7} x-41387.9$ & 0.99972 & $0.5-146.3$ & 0.002 & 0.010 \\
\hline Thymidine & $Y=5.06990 \times 10^{7} x-9208.8$ & 0.99995 & $0.5-147.8$ & 0.015 & 0.100 \\
\hline Adenosine & $Y=6.75044 \times 10^{7} x+13235.0$ & 0.99992 & $0.5-149.3$ & 0.010 & 0.050 \\
\hline 2-deoxyadenosine & $Y=7.93610 \times 10^{7} x+19809.4$ & 0.99991 & $0.5-151.5$ & 0.002 & 0.010 \\
\hline Cordycepin & $Y=7.30463 \times 10^{7} x-2626.2$ & 0.99991 & $0.5-150.0$ & 0.010 & 0.050 \\
\hline
\end{tabular}

Table 2. Precision, repeatability, stability and recovery of nine analytes.

\begin{tabular}{cccccc}
\hline \multirow{2}{*}{ Analyte } & \multicolumn{2}{c}{ Precision $(\mathrm{RSD}, \mathbf{\%}, \boldsymbol{n = 6 )}$} & \multirow{2}{*}{$\begin{array}{c}\text { Stability } \\
\text { (RSD, \%, } \boldsymbol{n}=\mathbf{6})\end{array}$} & \multicolumn{2}{c}{ Recovery $\mathbf{( \% , \boldsymbol { n } = \mathbf { 3 } )}$} \\
\cline { 2 - 3 } & Intraday & Interday & & Mean & RSD (\%) \\
\hline Uracil & 1.64 & 2.21 & 2.05 & 96.9 & 5.81 \\
Uridine & 1.21 & 1.94 & 1.54 & 100.6 & 4.78 \\
Inosine & 1.00 & 2.29 & 2.25 & 79.4 & 3.68 \\
Guanosine & 2.12 & 1.75 & 1.88 & 80.6 & 4.71 \\
Adenine & 0.96 & 2.32 & 2.29 & 80.6 & 2.93 \\
Thymidine & 1.11 & 1.60 & 1.76 & 102.7 & 1.32 \\
Adenosine & 1.29 & 2.61 & 1.71 & 103.1 & 4.38 \\
2-deoxyadenosine & 1.68 & 1.49 & 0.59 & 101.5 & 2.75 \\
Cordycepin & 1.64 & 2.80 & 1.63 & 90.4 & 2.88 \\
\hline
\end{tabular}




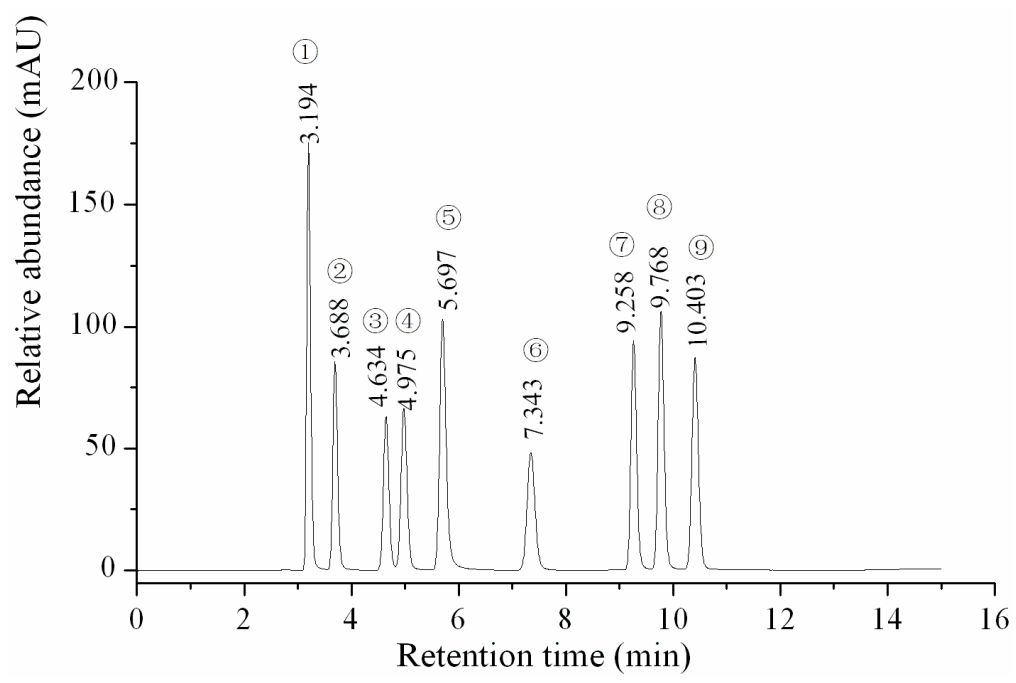

Figure 1. HPLC profile of the standard samples. (1) Uracil; (2) Uridine; (3) Inosine; (4) Guanosine; (5) Adenine; (6) Thymidine; (7) Adenosine; (8) 2-deoxyadenosine; (9) Cordycepin.

The retention time repeatability of nine nucleosides and nucleobases were tested on seven consecutive days. The result showed that the recoveries were $96.9 \%, 100.6 \%, 79.4 \%, 80.6 \%, 102.7 \%$, $103.1 \%, 80.6 \%, 101.5 \%$, and $90.4 \%$ for uracil, uridine, inosine, guanosine, Thymidine, adenosine, adenine, 2-deoxyadenosine and cordycepin, respectively.

\subsection{Contents of the Nucleosides and Nucleobases in Different Tissues}

The contents of seven nucleosides (cordycepin, adenosine, guanosine, uridine, inosine, thymidine and 2-deoxyadenosine) and two nucleobases (adenine and uracil) in five different materials are shown in Figure 2 and Table 3. Adenosine was not detected in the cadaver of Phassus nodus, and the content of adenosine in the natural fruit body $(761.5 \pm 56.4 \mathrm{mg} / \mathrm{kg})$ and cultured stroma $(721.2 \pm 31.8 \mathrm{mg} / \mathrm{kg})$ was significantly higher than in the cultured mycelium $(623.9 \pm 15.4 \mathrm{mg} / \mathrm{kg})$ and cultured coremium $(582.9 \pm 42.6 \mathrm{mg} / \mathrm{kg})$. The relative concentrations of uracil, uridine, inosine, guanosine, adenine, thymidine and 2-deoxyadenosine in the different tissues were compared. Thymidine and 2-deoxyadenosine only were found in the cadaver of Phassus nodus and the cultured mycelium, respectively. Inosine only was detected in the natural fruit body and the cadaver of Phassus nodus. The content of adenine in the cultured coremium was $186.6 \pm 16.6 \mathrm{mg} / \mathrm{kg}$, which was much higher than the other four samples. The highest concentrations of guanosine and uridine were found in the cultured mycelium, which contained $688.9 \pm 14.8 \mathrm{mg} / \mathrm{kg}$ and $1396.6 \pm 49.1 \mathrm{mg} / \mathrm{kg}$, respectively. The content of uracil in the cultured stroma was $138.1 \pm 4.8 \mathrm{mg} / \mathrm{kg}$, which was slightly higher than the other four materials. With the exception of the adenosine content, the cultured mycelium contained amounts of adenine, guanosine, uridine and uracil that were significantly higher than the values in the natural O. xuefengensis. The contents of adenine, guanosine and uracil in the cultured stroma were also much higher than in the natural O. xuefengensis. Therefore, the cultured O. xuefengensis mycelium and stroma are possibly helpful to further research on pharmacological activity of O. xuefengensis, and might have a promising application in health and functional product development. 


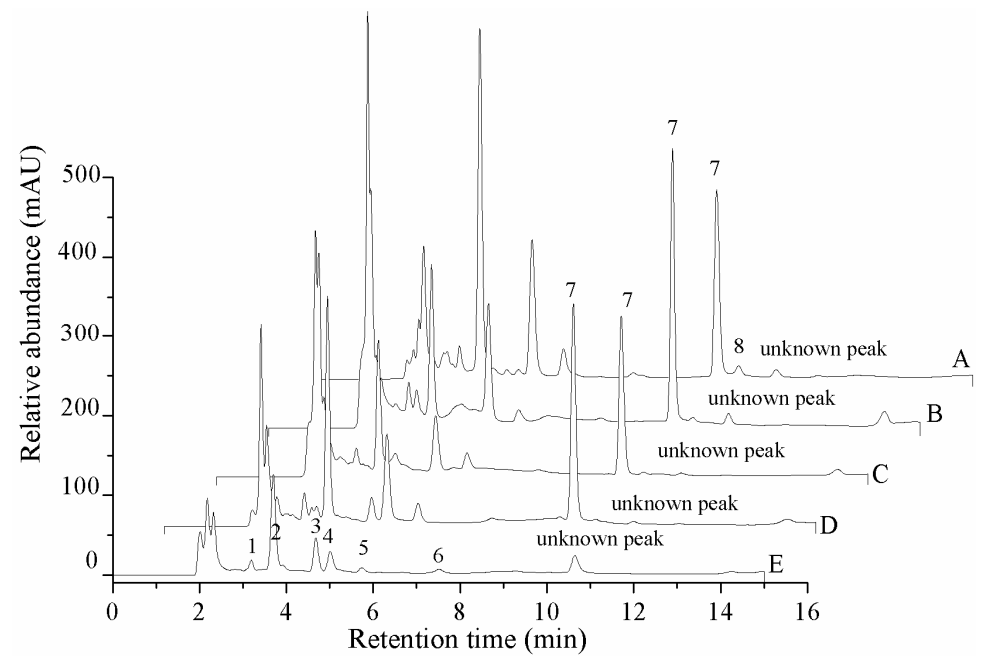

Figure 2. HPLC profile of the extraction of the nucleosides and nucleobases of O. xuefengensis. A. Cultured mycelium; B. Cultured stroma; C. Cultured coremium; D. Natural fruit body; E. Cadaver of phassus nodus. 1. Uracil; 2. Uridine; 3. Inosine; 4. Guanosine; 5. Adenine; 6. Thymidine; 7. Adenosine; 8. 2-deoxyadenosine.

Table 3. Contents (mg/kg) of nine nucleosides and nucleobases in different tissues of O. xuefengensis.

\begin{tabular}{|c|c|c|c|c|c|}
\hline Analyte & $\begin{array}{l}\text { Natural Fruit } \\
\text { Body }\end{array}$ & $\begin{array}{c}\text { Cadaver of } \\
\text { Phasus Nodus }\end{array}$ & $\begin{array}{l}\text { Cultured } \\
\text { Mycelium }\end{array}$ & $\begin{array}{l}\text { Cultured } \\
\text { Stroma }\end{array}$ & $\begin{array}{l}\text { Cultured } \\
\text { Coremium }\end{array}$ \\
\hline Cordycepin & nd & nd & nd & nd & nd \\
\hline 2-deoxyriboside & nd & nd & $62.8 \pm 4.9$ & nd & nd \\
\hline Adenosine & $761.5 \pm 56.4 \mathrm{aA}$ & nd & $623.9 \pm 15.4 \mathrm{bB}$ & $721.2 \pm 31.8 \mathrm{aAB}$ & $582.9 \pm 42.6 \mathrm{bB}$ \\
\hline Thymidine & nd & $66.8 \pm 6.6$ & nd & nd & nd \\
\hline Ade & $83.7 \pm 8.9 \mathrm{cBC}$ & $34.8 \pm 0.8 \mathrm{dC}$ & $126.1 \pm 9.1 \mathrm{bB}$ & $155.9 \pm 12.2 \mathrm{abAB}$ & $186.6 \pm 16.6 \mathrm{aA}$ \\
\hline Guanosine & $513.6 \pm 17.9 \mathrm{cC}$ & $179.7 \pm 5.4 \mathrm{dD}$ & $688.9 \pm 14.8 \mathrm{aA}$ & $626.1 \pm 24.8 \mathrm{bB}$ & $502.1 \pm 11.5 \mathrm{cC}$ \\
\hline Inosine & $177.6 \pm 0.9 \mathrm{bB}$ & $207.6 \pm 2.5 \mathrm{aA}$ & nd & nd & nd \\
\hline Uridine & $975.2 \pm 41.6 \mathrm{bB}$ & $506.9 \pm 8.1 \mathrm{cC}$ & $\begin{array}{c}1396.6 \pm \\
49.1 \mathrm{aA}\end{array}$ & $869.7 \pm 36.0 \mathrm{bB}$ & $995.2 \pm 85.6 \mathrm{bB}$ \\
\hline Uracil & $69.2 \pm 2.7 c C$ & $39.4 \pm 0.4 \mathrm{eE}$ & $87.1 \pm 6.9 \mathrm{bB}$ & $138.1 \pm 4.8 \mathrm{aA}$ & $55.9 \pm 1.2 \mathrm{dD}$ \\
\hline
\end{tabular}

a Means \pm standard deviation $(n=3)$ with different lowercase and uppercase letters indicate significant difference at the 0.05 level and extremely significant difference at the 0.01 level, by multiple range test, respectively.

The contents of adenosine in the natural stroma and cultured mycelium, stroma, and coremium of O. xuefengensis were $760,620,720$ and $580 \mathrm{mg} / \mathrm{kg}$, respectively, which was slightly lower than natural O. sinensis $(680-960 \mathrm{mg} / \mathrm{kg}$ ) reported by Zhao et al. and Wang et al. [12,13], and was significantly higher than O. sinensis (46.4-480.2 mg/ $\mathrm{kg}$ ), as reported by Xie et al. Zong et al. and Fan et al. [14-16]. The adenine content in the three cultured samples, were $126.1,155.9$ and $186.6 \mathrm{mg} / \mathrm{kg}$, respectively, which were higher than those measured for natural O. sinensis $(4.4-120 \mathrm{mg} / \mathrm{kg})$ [12-16]. There were no significant difference in content of uridine between $O$. xuefengensis and natural $O$. sinensis. The content of guanosine in both the natural and cultured O. xuefengensis $(179.7-688.9 \mathrm{mg} / \mathrm{kg}$ ) was lower than the value $(1011-1670 \mathrm{mg} / \mathrm{g}$ ) reported in natural O. sinensis by Wang et al. and Zong et al. [15,17]. Three cultured samples of $O$. xuefengensis and natural $O$. sinensis, reported by Zong et al. [17], displayed roughly the same amounts of guanosine, which were $502.1-688.9 \mathrm{mg} / \mathrm{kg}$ and $484-832 \mathrm{mg} / \mathrm{kg}$, respectively. These data illustrated that the cultured O. xuefengensis mycelium and stroma might be a promising substitute for natural $O$. sinensis.

\subsection{Identification of Cordycepin}

In our experiment, the chromatograms of the natural and cultured samples all showed peaks at $10.67 \pm 0.05 \mathrm{~min}$ after the expected retention times for cordycepin $(10.43 \pm 0.08 \mathrm{~min})$, and the same 
peaks at $10.69 \pm 0.04$ min were found in natural O. sinensis (Figure 3). To verify whether O. xuefengensis contained cordycepin, water extracts of $O$. xuefengensis and O. sinensis were analysed with ESI/TOF-MS. The mass spectrum of cordycepin showed the main peak at $m / z=252.1$, corresponding to the protonated molecular ion, and a peak at $m / z=249.8$, corresponding to the deprotonation molecular ion (Figure 4A,B). However, as Figure 4C,D display, the mass spectrum of the LC peak at $10.67 \pm 0.05 \mathrm{~min}$ of $O$. xuefengensis had no signal at $m / z=252.1$ in positive mode and no signal at $m / z=249.8$ in negative ion mode, which demonstrated that the LC peak at $10.67 \pm 0.05 \mathrm{~min}$ of $O$. xuefengensis was not the absorbance peak of cordycepin. The mass spectrum of the LC peak at $10.69 \pm 0.04 \mathrm{~min}$ of natural O. sinensis is shown in Figure $4 \mathrm{E}$, with weak signal at $m / z=252.1$, indicating the protonated species, but no signal was detected at $m / z=249.8$ in negative ion mode (Figure $4 \mathrm{~F}$ ). This may be the reason for the controversy over whether $O$. sinensis contained cordycepin $[3,15,18]$. In our experiment, cordycepin was not detected in either O. sinensis or O. xuefengensis. However, cordycepin in the natural C. sinensis is confirmed in other reports. In addition, the same unknown LC peaks were also reported in Cordyceps gunnni and C. taii [2,18], which is likely to be a feature peak in Ophiocordyceps, and the identification of the unknown peak requires further research.

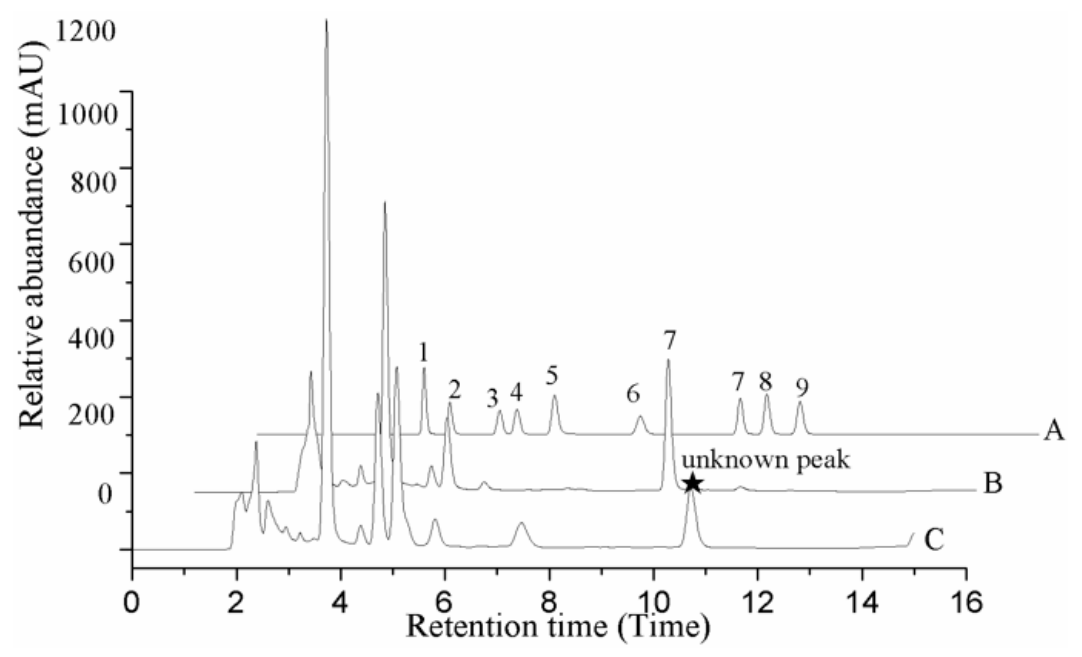

Figure 3. HPLC profile of O. sinensis. A. Standard samples. B. Natural fruit body. C. Cadaver of Hepialidae. 1. Uracil; 2. Uridine; 3. Inosine; 4. Guanosine; 5. Adenine; 6. Thymidine; 7. Adenosine; 8. 2-deoxyadenosine; 9. Cordycepin.

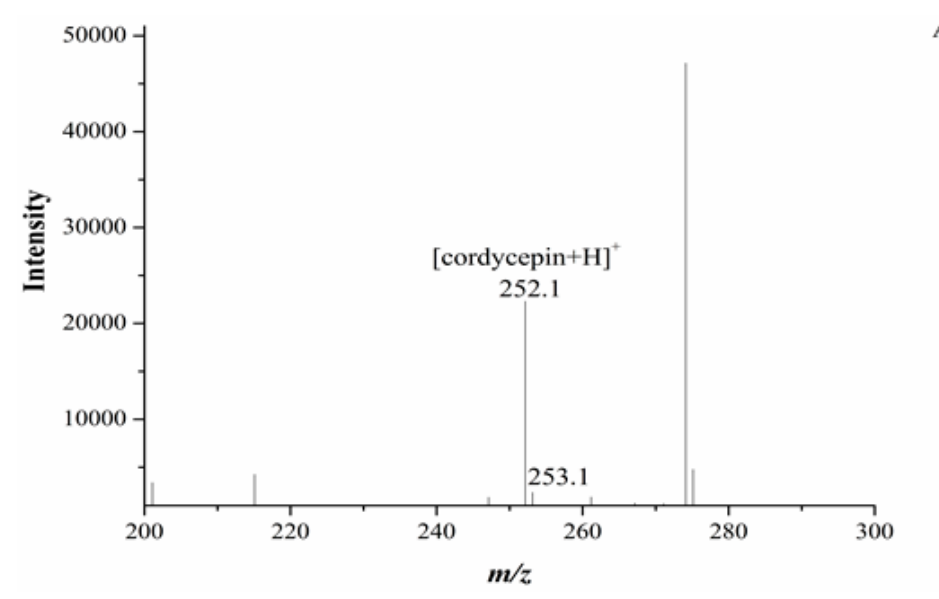

Figure 4. Cont. 

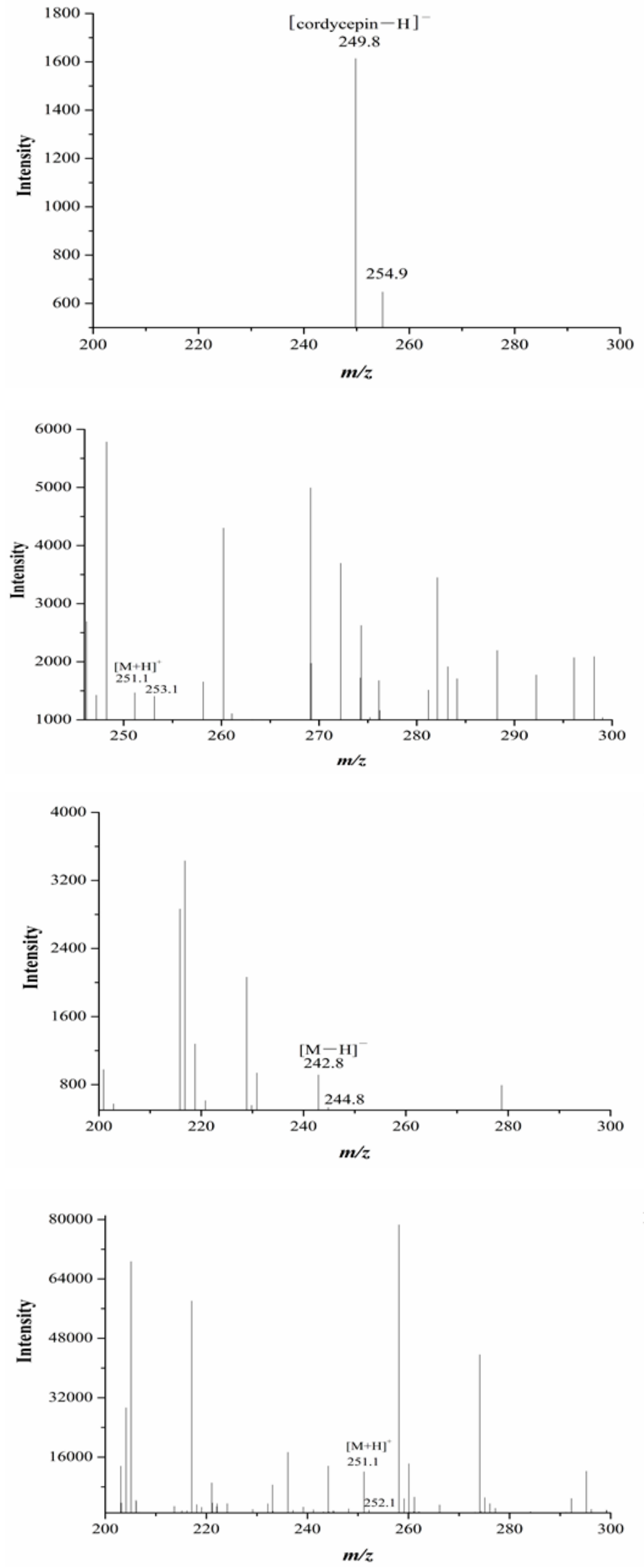

Figure 4. Cont. 


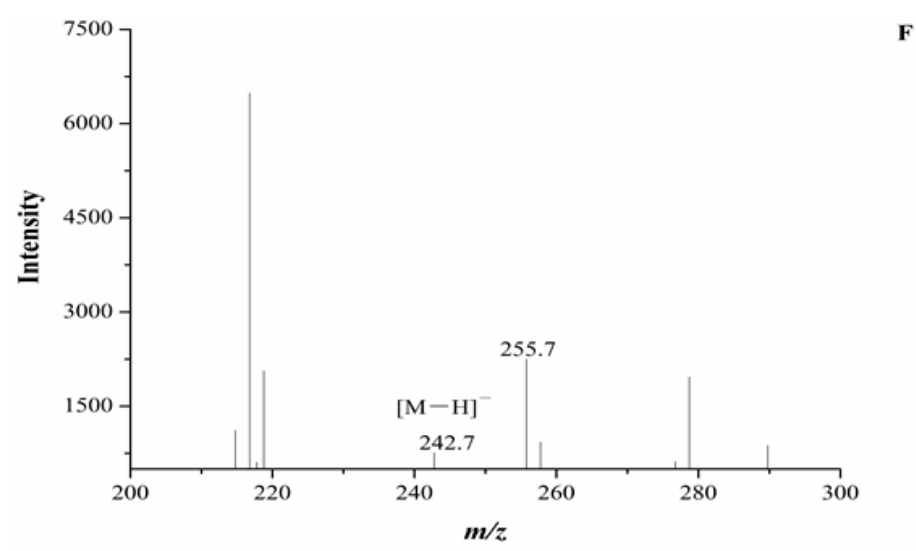

Figure 4. ESI/TOF-MS spectra of cordycepin, O. xuefengensis and O. sinensis in positive and negative ion modes. (A) Cordycepin in positive ion mode; (B) Cordycepin in negative ion mode; (C) The LC peaks O. xuefengensis in positive ion mode; (D) The LC peaks O. xuefengensis in negative ion mode; (E) The LC peaks of $O$. sinensis in positive ion mode; (F) The LC peaks of $O$. sinensis in negative ion mode.

\section{Experimental}

\subsection{Materials}

The fresh stroma of natural O. xuefengensis (Figure 5A), named MHHNU7966-WS (GenBank accession number KY454625), was collected from Phassus nodus in the living root of Clerodendrum cyrtophyllum in the Xuefeng Mountains of the Hunan Province in South China. The fungal strain, named MHHNU7966-IS (GenBank accession number KU356800), was isolated from the fresh stroma of natural O. xuefengensis. The fungus bearing stroma of O. xuefengensis (Figure 5B) was cultivated in artificial media and collected. The dried fruiting bodies of natural O. sinensis were purchased from Qinghai Spring Medicinal Resources Technology Co., Ltd., Xining, Qinghai, China.

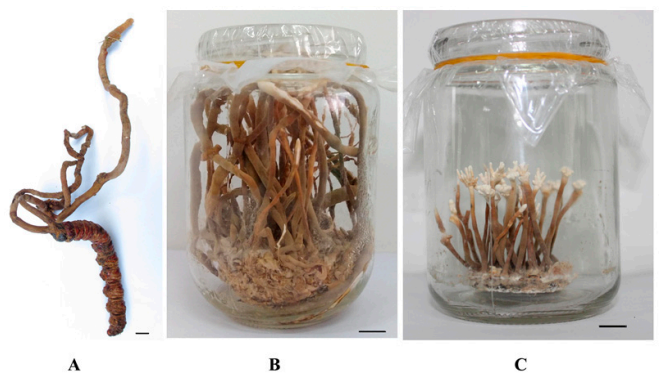

Figure 5. Ophiocordyceps xuefengensis. (A) Natural O. xuefengensis; (B) Cultured stroma; (C) Cultured coremium. Scale bars: $10 \mathrm{~mm}$.

\subsection{Inoculum Preparation and Flask Cultures}

The medium for agar slant consisted of the following (g/L): potato infusion, 200; glucose, 10; peptone, 10; pupa powder, 10; and agar, 20.0. The production medium contained the following (g/L): potato infusion, 200; sucrose,15; peptone, 10; $\mathrm{Na}_{2} \mathrm{HPO}_{4}, 1.18 ; \mathrm{KH}_{2} \mathrm{PO}_{4}, 1.13 ; \mathrm{MgSO}_{4} \cdot 7 \mathrm{H}_{2} \mathrm{O}, 3 ; \mathrm{KNO}_{3}, 3$; Vitamin $\mathrm{B}_{1}, 0.006$; Vitamin $\mathrm{B}_{2}, 0.003$; Vitamin $\mathrm{B}_{6}, 0.0004$; and nicotinamide, 0.02 . The $\mathrm{pH}$ values of the media were all adjusted to $6.5 \mathrm{by} \mathrm{HCl}$ or $\mathrm{NaOH}$. For the seed preparation, the mycelium of strain MHHNU7966-IS was picked from an agar slant culture, placed into $50 \mathrm{~mL}$ of sterile seed medium in a $250 \mathrm{~mL}$ Erlenmeyer flask, and incubated at $25^{\circ} \mathrm{C}$ with shaking at $150 \mathrm{rpm}$ for 5 days. The production medium in a $250 \mathrm{~mL}$ flask was inoculated with $5 \%(\mathrm{v} / \mathrm{v})$ of the seed culture and was incubated at $25^{\circ} \mathrm{C}$ and $140 \mathrm{rpm}$ for 7 days. The mycelium was collected by centrifugation at $6000 \times g$ for $15 \mathrm{~min}$. 


\subsection{Artificial Cultivation of Coremium}

The solid medium consisting of the following (g/bottle): rice, 10; oat, 5; millet, 2.5; pupa powder, $0.5 ; \mathrm{CaCO}_{3}, 0.1$; and $\mathrm{CaSO}_{4} \cdot 2 \mathrm{H}_{2} \mathrm{O}, 0.1$, as well as the nutrient solution $(30 \mathrm{~mL})$, which contained the following $(\mathrm{g} / \mathrm{L})$ : potato infusion, 200; sugar,10; peptone, 5; $\mathrm{Na}_{2} \mathrm{HPO}_{4}, 1.18 ; \mathrm{KH}_{2} \mathrm{PO}_{4}$, $1.13 ; \mathrm{MgSO}_{4} \cdot 7 \mathrm{H}_{2} \mathrm{O}, 1.5$; and $\mathrm{KNO}_{3}$, 2, were poured into a $350 \mathrm{~mL}$ glass jar, mixed with slow oscillations, incubated at $50{ }^{\circ} \mathrm{C}$ for $2 \mathrm{~h}$, and then sterilized in high-pressure steam at $121^{\circ} \mathrm{C}$ for $40 \mathrm{~min}$. The sterilized solid medium in a 350-mL glass jar was inoculated with $2 \mathrm{~mL}$ of the seed culture and was incubated for $30 \mathrm{~d}$ at $25^{\circ} \mathrm{C}$ at $60 \%$ relative humidity, in complete darkness. The solid medium, completely covered by mycelium, was used as coremium development. For this the solid medium was incubated with the normal day-night cycle (light intensity was $100-200 \mathrm{~lx}$ during the day) at $28^{\circ} \mathrm{C}$ for $60 \mathrm{~d}$. The coremium of O. xuefengensis was obtained in artificial media (Figure 5C).

\subsection{Extraction of the Nucleoside Analogues and Nucleobases}

All the samples were lyophilized to a constant weight and were ground by hand with a mortar and pestle. Samples $(0.2 \mathrm{~g})$ of the ground materials (two different natural tissues and three cultured development stages) were weighed accurately into individual test tubes and were extracted with $2 \mathrm{~mL}$ of $15 \%$ methanol (prepared with doubly distilled water) at $4{ }^{\circ} \mathrm{C}$ for $12 \mathrm{~h}$. Following ultrasonic extraction for $30 \mathrm{~min}$ and, then, centrifugation at $4000 \times g$ for $15 \mathrm{~min}$, the supernatant was decanted and retained, and the residue was resuspended in $2 \mathrm{~mL}$ of $15 \%$ methanol and extracted again as described above. The two supernatants were combined and filtered through a micropore filter membrane $(0.22 \mu \mathrm{m})$ for the high-performance liquid chromatography (HPLC) analysis. The extraction was donein triplicate for each tissue sample, and the mean value was determined.

\subsection{Standard Samples and the Linear Regression Equation}

Standard samples of adenosine, guanosine, uridine, inosine, thymidine, 2-deoxyadenosine adenine, uracil (Sigma-Aldrich, St. Louis, MO, USA), and cordycepin (Chinese Materials Research Centre, Beijing, China) were dissolved in doubly distilled water. The chemical structures of these reference compounds are shown in Figure 6. The determination of the linearity, the correlation coefficient, the reproducibility and the recovery of the nine investigated components were determined. The calibration curve (peak area ratio of the analytes versus the concentration of the analyte, $\mu \mathrm{g} / \mathrm{mL}$ ) over the studied range of the nine investigated components were calculated. The nucleosides and nucleobases in the samples were identified by comparing the retention times and spiking the Cordyceps samples with the standard solutions. The concentrations were calculated using the peak areas of the reference compounds.

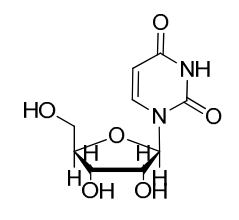

Uridine

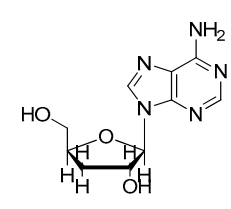

Cordycepin<smiles></smiles>

Uracil

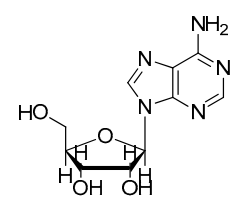

Adenosine

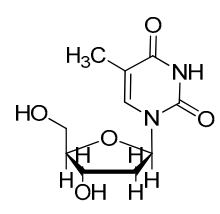

Thymidine

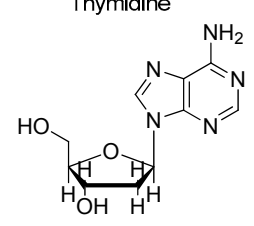

2'-Deoxyadenosine

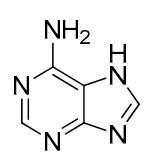

adenine
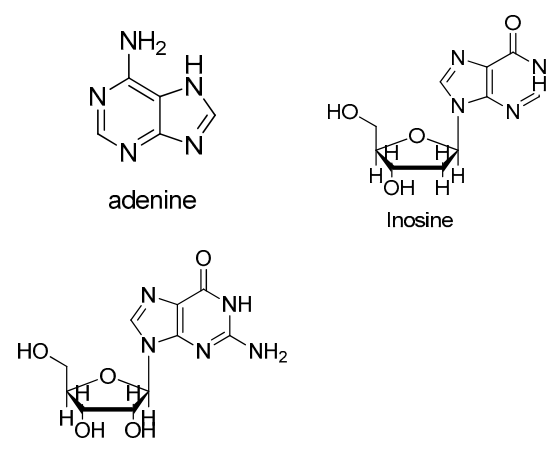

Guanosine

Figure 6. Structures of uridine, uracil, thymidine, adenine, inosine, cordycepin, adenosine, $2^{\prime}$-deoxyadenosine and guanosine. 


\subsection{HPLC Analysis}

The nucleoside analogues and nucleobases were determined by reverse phase HPLC using a Shimadzu Series LC-20 AT high-performance liquid chromatograph (HPLC) (Shimadzu, Kyoto, Japan) equipped with a binary high-pressure pump, and a SPD-20AV UV-Vis detector connected to LC solution software (Shimadzu). Separation was carried out in a pre-packed Diamonsil C18 reverse phase HPLC column $(250 \mathrm{~mm} \times 4.6 \mathrm{~mm}$, particle size $5 \mu \mathrm{m}$; Dikma Technologies Incorporation, Lake Forest, CA, USA). The absorbance of the eluates was monitored at $260 \mathrm{~nm}$. The mobile phase and elution profile were analysed as described by Xiao et al. [18,19]. The mobile phase was comprised of (A) ultrapure water and (B) methanol. All the solutions were degassed by sonication prior to use. The elution conditions were as follows 0-3 min, isocratic 15\% B; 3.0-3.5 min, linear gradient 15-23\% $\mathrm{B}$; 3.5-8.5 min, isocratic $23 \% \mathrm{~B}$; 8.5-9.0 min, linear gradient 23-35\% B; and 9.0-15 min, isocratic 35\% $\mathrm{B}$, with a final column flush with $100 \% \mathrm{~B}$ for $40 \mathrm{~min}$ before reconditioning the column with $15 \% \mathrm{~B}$ isocratic for $30 \mathrm{~min}$, all at $30{ }^{\circ} \mathrm{C}$. The flow-rate was $1 \mathrm{~mL} / \mathrm{min}$, and the injection volume was $20 \mu \mathrm{L}$. All the injections were repeated three times to ensure reproducibility. Nucleosides were identified by comparing the retention time, purity coefficient and spectrum against known chemical standards. The external standard method was used for nucleoside determinations.

\subsection{ESI/TOF-MS Analysis}

For the analysis with the electrospray ionization/time-of-flight mass spectrometer (ESI/TOF-MS) (PerkinElmer, New Orleans, LA, USA), the source voltage, capillary exit voltage, Syringe pump flow rate, drying gas temperature, drying gas rate, spray gas pressure, and evaporator temperature were set to $4.0 \mathrm{kV}, 100 \mathrm{~V}, 10 \mu \mathrm{L} / \mathrm{min}, 385^{\circ} \mathrm{C}, 12 \mu \mathrm{L} / \mathrm{min}, 80 \mathrm{psi}$, and $385^{\circ} \mathrm{C}$, respectively. The samples were analysed in both positive and negative ion modes. The mass-selective detector was manually tuned using a dual nebulizer electrospray source with an internal calibrant consisting of an unknown fluorinated compound delivery system, which introduced a constant flow $(10 \mu \mathrm{L} / \mathrm{min})$ of calibrating solution containing the internal reference masses $(m / z 118.0863,322.0481)$ in positive ion mode. Tuning was conducted over the range of $m / z 100-300$.

\section{Conclusions}

In this research, the nucleosides content and distribution in five different samples of O. xuefengensis (two different natural tissues and three cultured development stages) were systematically analysed by reverse phase HPLC. Currently, nucleosides are believed to be the active components in Cordyceps. Seven main nucleosides (cordycepin, adenosine, guanosine, uridine, inosine, thymidine and 2-deoxyadenosine) and two nucleobases (adenineand uracil) were determined in this study. The results showed that the natural fruit body contained the highest amount of adenosine $(761.5 \pm 56.4 \mathrm{mg} / \mathrm{kg})$, followed by the cultured stroma $(721.2 \pm 31.8 \mathrm{mg} / \mathrm{kg})$ and cultured mycelium $(623.9 \pm 15.4 \mathrm{mg} / \mathrm{kg})$, whereas the cadaver of Phassus nodus had almost no adenosine content. Inosine was only detected in the natural fruit body and the cadaver of Phassus nodus. The adenine, guanosine, uridine and uracil contents were significantly higher in the cultured mycelium than in the natural samples. The natural and cultured O. xuefengensis, as well as the reported natural O. sinensis by Wang et al. [13], contained roughly the same amounts of adenosine. The cultured $O$. xuefengensis had the higher guanosine concentration than the natural O. xuefengensis.

The yield of wild O. xuefengensis has been declining in recent years because of its strict growing habitat and excessive harvesting. In addition, the gathering of wild O. xuefengensis requires digging out the living root of the medicinal plant Clerodendrum cyrtophyllum and cutting open the living root, which leads to damage of the medicinal plant C. cyrtophyllum. The cultured O. xuefengensis mycelium and stroma may be a promising substitute for natural O. xuefengensis, as well as a means forprotecting the natural resource. 
Acknowledgments: This work was financially supported by the Science and Technology Major Project of Hunan Province, China (grant No. 2014FJ1007).

Author Contributions: Z.-H.C. and J.Z. contributed to the conception of the study; J.Z., L.W. Contributed to experiment and analysis of the nucleosides and nucleobases; Z.-M.H. and P.Z. performed the cultivation of O. xuefengensis; J.Z. and Z.-H.C. wrote the manuscript. All authors read and approved the manuscript.

Conflicts of Interest: The authors declare no conflicts of interest.

\section{References}

1. Galmarini, C.M.; Mackey, J.R.; Dumontet, C. Nucleoside analogues and nucleobases in cancer treatment. Lancet Oncol. 2002, 3, 415-424. [CrossRef]

2. Xiao, J.H.; Qi, Y.; Xiong, Q. Nucleosides, a valuable chemical marker for quality control in traditional Chinese medicine Cordyceps. Recent Pat. Biotechnol. 2013, 7, 153-166. [CrossRef] [PubMed]

3. Li, S.P.; Yang, F.Q.; Tsim, K.W. Quality control of Cordyceps sinensis, a valued traditional Chinese medicine. J. Pharm. Biomed. Anal. 2006, 41, 1571-1584. [CrossRef] [PubMed]

4. Chen, P.X.; Wang, S.; Nie, S.; Marcone, M. Properties of Cordyceps sinensis: A review. J. Funct. Foods 2013, 5, 550-569. [CrossRef]

5. Tuli, H.S.; Sharma, A.K.; Sandhu, S.S.; Kashyap, D. Cordycepin: A bioactive metabolite with therapeutic potential. Life Sci. 2013, 93, 863-869. [CrossRef] [PubMed]

6. Zhou, X.; Luo, L.; Dressel, W.; Shadier, G.; Krumbiegel, D.; Schmidtke, P.; Zepp, F.; Meyer, C.U. Cordycepin is an immunoregulatory active ingredient of Cordyceps sinensis. Am. J. Chin. Med. 2008, 36, 967-980. [CrossRef] [PubMed]

7. Jeong, M.H.; Lee, C.M.; Lee, S.W.; Seo, S.Y.; Seo, M.J.; Kang, B.W.; Jeong, Y.K.; Choi, Y.J.; Yang, K.M.; Jo, W.S. Cordycepin-enriched Cordyceps militaris induces immunomodulation and tumor growth delay in mouse-derived breast cancer. Oncol. Rep. 2013, 30, 1996-2002. [CrossRef] [PubMed]

8. Pharmacopoeia Commission of PRC. Pharmacopoeia of the People's Republic of China, vol. I; Chemical Industry Press: Beijing, China, 2005; p. 75.

9. Wen, T.C.; Zhu, R.C.; Kang, J.C.; Huang, M.H. Ophiocordyceps xuefengensis sp. nov. from larvae of Phassus nodus (Hepialidae) in Hunan Province, southern China. Phytotaxa 2013, 123, 41-50.

10. Zheng, B.; Xie, F.Y.; Cai, G.H.; Zhu, R.C.; Li, K.; Gao, S.Q.; Tan, D.B.; Hao, X.Y.; Qin, Y.H. Effects of Ophiocordyceps xuefengensis on proliferation of DC-CIK cells and activity of killing HepG-2 cells by DC-CIK cells. Chin. J. Immunol. 2015, 31, 189-192.

11. Zou, J.; Wu, L.; He, Z.M.; Zhang, P.; Chen, Z.H. Biological characteristics and cultivation of Ophiocordyceps xuefengensis. Mycosystema 2017, 36, 1104-1110.

12. Zhao, H.Q.; Wang, X.; Li, H.M.; Yang, B.; Yang, H.J.; Huang, L. Characterization of nucleosides and nucleobases in natural Cordyceps by HILIC-ESI/TOF/MS and HILIC-ESI/MS. Molecules 2013, 18, 9755-9769. [CrossRef] [PubMed]

13. Wang, J.Q.; Kan, L.J.; Nie, S.P.; Chen, H.H.; Cui, S.W.; Phillips, A.O.; Phillips, G.O.; Li, Y.J.; Xie, M.Y. A comparison of chemical composition, bioactive components and antioxidant activity of natural and cultured Cordyceps sinensis. LWT-Food Sci. Technol. 2015, 63, 2-7. [CrossRef]

14. Xie, J.; Huang, L.; Hu, W.; He, Y.; Wong, K. Analysis of the main nucleosides in Cordyceps sinensis by LC/ESI-MS. Molecules 2010, 15, 305-314. [CrossRef] [PubMed]

15. Zong, S.Y.; Han, H.; Wang, B.; Li, N.; Dong, T.T.; Zhang, T.; Tsim, K.W. Fast simultaneous determination of 13 nucleosides and nucleobases in Cordyceps sinensis by UHPLC-ESI-MS/MS. Molecules 2015, 20, 21816-21825. [CrossRef] [PubMed]

16. Fan, H.; Li, S.P.; Xiang, J.J.; Lai, C.M.; Yang, F.Q.; Gao, J.L.; Wang, Y.T. Qualitative and quantitative determination of nucleosides, bases and their analogues in natural and cultured Cordyceps by pressurized liquid extraction and high performance liquid chromatography-electrospray ionization tandem mass spectrometry HPLC-ESI-MS/MS). Anal. Chim. Acta 2006, 567, 218-228.

17. Zeng, W.B.; Yu, H.; Ge, F.; Yang, J.Y.; Chen, Z.H.; Wang, Y.B.; Dai, Y.D.; Adams, A. Distribution of nucleosides in populations of Cordyceps cicadae. Molecules 2014, 19, 6123-6141. [CrossRef] [PubMed]

18. Xiao, J.H.; Xiao, D.M.; Sun, Z.H.; Xiong, Q.; Liang, Z.Q.; Zhong, J.J. Chemical compositions and antimicrobial property of three edible and medicinal Cordyceps species. J. Food Agric. Environ. 2009, 7, 91-100. 
19. Xiao, J.H. Cordyceps spp. Fermentation, Its Metabolites Separation and Their Bioactivity Study; East China University of Science and Technology: Shanghai, China, 2010; pp. 83-88.

Sample Availability: Samples of the Ophiocordyceps xuefengensis are available from the authors. article distributed under the terms and conditions of the Creative Commons Attribution (CC BY) license (http:/ / creativecommons.org/licenses/by/4.0/). 